\title{
Methods of Alignment for On-Line Signature Verification
}

\author{
Xinghua Xia \\ School of Information and Control Engineering \\ Shenyang JianZhu University \\ Shenyang, China \\ e-mail: xxh8787@sjzu.edu.cn \\ Fangjun Luan \\ School of Information and Control Engineering \\ Shenyang JianZhu University \\ Shenyang, China \\ e-mail: luanfangjun@sjzu.edu.cn
}

\author{
Shoujin Wang \\ School of Information and Control Engineering \\ Shenyang JianZhu University \\ Shenyang, China \\ e-mail: wangshoujin@sjzu.edu.cn \\ Dafang Yang \\ School of Information and Control Engineering \\ Shenyang JianZhu University \\ Shenyang, China \\ e-mail: yangdafang@sjzu.edu.cn
}

\begin{abstract}
Size, location and rotation angle of on-line handwritten signature will have effects on the performance of verification. Methods of alignment for online signature verification are proposed in this paper, the size, location and rotation angle of test and reference signatures are aligned effectively to reduce the variations of different inputting. A method of SCLC-DTW with signature curves location constraint is proposed also to improved efficiency of similarity calculation. Several experiments are carried out on standard on-line signature dataset DB1, which consists of $\mathbf{5 0 0 0}$ signatures from 100 individuals in total. The best result is given by $E E R=2.99 \%$, which indicates the effectiveness of our proposed methods.
\end{abstract}

Keywords-on-line signature verification; alignment;DTW; SCLC-DTW

\section{INTRODUCTION}

On-line handwritten signature verification is one of the most acceptable biometrics due to the fact that online handwritten signatures have long been established as most widespread means of personal verification. The signatures are difficult to be imitated and forged because of unique and consistent for a given period. Experimental results presented in great many research works have indicated that accuracy of on-line signature verification is not lower than other biometrics [1-2].

On-line handwritten signatures are collected through by the user writing on the collective devices in real-time, and the signatures are presented by time series. By the reasons of collective devices, external environments and psychological factors, there are variation of the size, location and rotation angle of signatures for each inputting. Furthermore, from the perspective of the kinematic, signatures are rapid and skilled human actions which mainly determined by the dynamics of muscle system. Signatures would not keeping higher consistent for a long time since external and internal environment exchanging. Thus, it is important to effectively align the test signatures to references, and reduce the influences the inconsistency of the size, location and rotation angle on the performances of online handwritten signature verification [3-7]. During online signature verification process, the authenticity of test signature is evaluated by matching its features against those stored in knowledge base for given individual. There are some commonly used verification methods, such as template matching methods, statistical based methods, and structural based methods [8-10].

In our works, we propose some methods of alignment to obtain the optimal matching between test signatures and references in order to improve the recognition rate. We also propose a method of similarity calculation based on DTW with signature curves location constraint to reduce the complexity of computation.

\section{ALIGNMENT FOR ON-LINE SIGNATURE}

To reduce the influences of inconsistency on performances of on-line signature verification, test signatures should be aligned to reference coordinate system before verification. The inconsistencies are mainly caused by variations of the size, location and rotation angle of signatures.

\section{A. Alignment with Size}

The size of signatures should be aligned due to the variation size of each inputting, and this variation would increase the dissimilarity between test signatures and references. The commonly used method of size alignment is max-min normalization to standardize the size of signature.

$$
\widehat{S}(n)=\frac{s(n)-S_{\min }}{S_{\max }-S_{\min }}
$$

Where, $\widehat{S}(n)=\{\hat{x}(n), \hat{y}(n)\}$ is normalized signature after size alignment, $S(n)=\{x(n), y(n)\}$ is original signature, $\quad \mathrm{S}_{\min }=\min _{\mathrm{n} \in\{1,2, \cdots, \mathrm{N}\}} \mathrm{S}(\mathrm{n}) \quad, \quad \mathrm{S}_{\max }=$ $\max _{n \in\{1,2, \cdots, N\}} S(n), N$ is total number of sampled points of signature. 


\section{B. Alignment of Signature Location}

As stated, the locations of signatures are different on the collective device at each time inputting, and this variation would also increase the dissimilarity between the test signatures and references. The locations of signatures should be also aligned before verification, and the signature location could be aligned by signature center and signature centroid respectively.

Locations alignment with signature center is given by

$\mathrm{S}_{\text {center }}{ }^{\prime}(\mathrm{n})=\mathrm{S}(\mathrm{n})-\mathrm{S}_{\text {center }}$

Where, $S_{\text {center }}{ }^{\prime}(n)$ is the signature after location alignment with signature center, $\mathrm{S}_{\text {center }}=\left(\mathrm{S}_{\max }-\mathrm{S}_{\min }\right) / 2$ is the signature center, $\mathrm{S}_{\min }$ and $S_{\max }$ are defined as above 。

Locations of signatures could be aligned with signature centroid is

$\mathrm{S}_{\text {centroid }}{ }^{\prime}(\mathrm{n})=\mathrm{S}(\mathrm{n})-\mathrm{S}_{\text {centroid }}$

Where, $S_{\text {centroid }}(n)$ is the signature after location alignment with signature centroid, $\mathrm{S}_{\text {centroid }}=$ $\frac{1}{N} \sum_{n=1}^{N} S(n)$ is the signature centroid.

\section{Alignment of Signature Rotation Angle}

The variability in orientation of signatures would produce noise in the stage of verification. The signature rotation angle is aligned by rotate the signature coordinate axes with a suitable reference angle to the standard reference coordinate system. The coordinate axes of signature would be rotated by means of a rotation matrix as bellow

$$
\begin{aligned}
& x^{\prime}=x \cdot \cos (\alpha)-y \cdot \sin (\alpha) \\
& y^{\prime}=x \cdot \sin (\alpha)-y \cdot \cos (\alpha)
\end{aligned}
$$

Where, $x$ and $y$ are the original coordinate, $x$ ' and $y$ ' are the new signature coordinate after rotation angle alignment, $\alpha$ is the reference rotate angle, which would be calculated as bellow

$\alpha=\frac{1}{2} \cdot \arctan \left(2 \cdot \mathrm{I}_{\mathrm{xycm}} /\left(\mathrm{I}_{\mathrm{ycm}}-\mathrm{I}_{\mathrm{xcm}}\right)\right)(6)$

where, $I_{x y c m}, I_{y c m}$ and $I_{x c m}$ are the moments of inertia referred to the reference centroid, which will be calculated as $\mathrm{I}_{\mathrm{xcm}}=\mathrm{I}_{\mathrm{x} 0}-\mathrm{Ny}_{\mathrm{cm}}^{2}, \mathrm{I}_{\mathrm{ycm}}=\mathrm{I}_{\mathrm{y} 0}-\mathrm{Nx}_{\mathrm{cm}}^{2}$ and $\mathrm{I}_{\mathrm{xycm}}=\mathrm{I}_{\mathrm{xy} 0}-\mathrm{Nx}_{\mathrm{cm}} \mathrm{y}_{\mathrm{cm}} \cdot \mathrm{I}_{\mathrm{x} 0}=\sum_{\mathrm{n}=1,2, \cdots, \mathrm{N}} \mathrm{y}^{2}(\mathrm{n})$, $\mathrm{I}_{\mathrm{y} 0}=\sum_{\mathrm{n}=1,2, \cdots, N} \mathrm{x}^{2}(\mathrm{n})$ and $\mathrm{I}_{\mathrm{xy} 0}=\sum_{\mathrm{n}=1,2, \cdots, N} \mathrm{x}(\mathrm{n}) \mathrm{y}(\mathrm{n})$.

\section{ON-LINE SIGNATURE VERIFICATION}

Dynamic time warping (DTW) is widely used in online signature verification when template matching approaches are considered. It is less efficient and more complexity of computation of traditional DTW if the number of sampled points were larger. A simple but efficient matching method based on complete information of signature is proposed.

For a given individual, let $\{\mathrm{s}(\mathrm{n})(\mathrm{x}, \mathrm{y}), \mathrm{n}=1,2, \ldots, \mathrm{N}\}$ and $\{\mathrm{t}(\mathrm{m})(\mathrm{x}, \mathrm{y}), \mathrm{m}=1,2, \ldots, \mathrm{M}\}$ be reference and test signature respectively, where, $\mathrm{N}$ and $\mathrm{M}$ denote the sampled points included in the two signatures; $(\mathrm{x}, \mathrm{y})$ denotes the curve of signature. The curves of reference and test signature are matched by DTW, and the optimal path is obtained by dynamic programming, i.e. $W_{(s, t),(x, y)}=\left\{w_{1}, w_{2}, \cdots, w_{r}, \cdots, w_{R}\right\} \quad$, where, $\max (N, M) \leq R \leq N+M-1 \quad, \quad w_{r}=\left(w_{r_{s}}, w_{r_{t}}\right)$ denotes the matching pair on the DTW path, which is called the signature curves location constraint of DTW matching. Considering the DTW matching with signature curves location constraint, the similarity of feature $F_{k}$ between reference and test signature is calculated by Euclidean distance, denotes as SCLCDTW.

$$
\begin{array}{r}
\operatorname{DIST}_{(s, t), F_{k}}=\mathrm{d}_{(s, t), F_{k}} \mid W_{(s, t),(x, y)}= \\
\sum_{r=1}^{R} \sqrt{\left(F_{s_{k}}\left(w_{r_{s}}\right)-F_{t_{k}}\left(w_{r_{t}}\right)\right)^{2}}
\end{array}
$$

Where, $\operatorname{DIST}_{(\mathrm{s}, \mathrm{t}), \mathrm{F}_{\mathrm{k}}}$ denotes the SCLC-DTW distance of feature $F_{k}$ between reference and the test signature, $\mathrm{W}_{(\mathrm{s}, \mathrm{t}),(\mathrm{x}, \mathrm{y})}$ denotes the optimal DTW matching path of signature curves between reference and test signature.

According to the discussions above, not only the complete information of signature but also the DTW dynamic programming is included in the proposed method of similarity calculation based on SCLC-DTW. Moreover, computational complexity of on-line signature verification is decreased by proposed method. The computational complexity of traditional DTW is given by $\mathrm{O}(\mathrm{K} * \mathrm{~N} * \mathrm{M})$, instead, the computational complexity of SCLC-DTW is given by $\mathrm{O}(\mathrm{N} * \mathrm{M}+\mathrm{K} *$ $(N+M-1)) \approx 0(N * M)$. It is more efficient of proposed SCLC-DTW than traditional DTW.

As for the verification, according to the threshold stored in knowledge base, we could output the verification result of genuine or forgery according to voting rule. The decision-making tag matrix of test signature is given as

$\operatorname{Vote}\left(s^{r e f}, k\right)=\left\{\begin{array}{l}1 \quad \text { if } \operatorname{DIST}_{(s, t), F_{k}} \leq \mathrm{TH}_{s} \text { ref },_{k} \\ 0 \quad \text { rule in others }\end{array}\right.$ $\sum_{1 \leq s} r e f_{\leq L_{s}, 1 \leq k \leq L_{c}} \operatorname{Vote}\left(s^{r e f}, k\right) \geq \operatorname{COUNT}_{\text {vote }}$, the test signature is genuine, otherwise, the test signature is forgery. Where, COUNTvote is the numbers of confirmed tag, Ls is the numbers of references, and $\mathrm{Lc}$ is the numbers of features used in verification. $\mathrm{TH}_{S}{ }{ } f_{{ }_{F}}$ is the decision threshold of feature $F_{k}$ for given individual reference $s^{\text {ref }}$.

\section{EXPERIMENT ATION}

\section{A. On-Line Signature Verification Dataset Description}

Several experiments based on large scale dataset of MCYT_Subcorpus_100 (DB1) [11] are carried out. DB1 consists of 5000 on-line signatures from 100 individuals. For each individual, there are 50 signatures in all, out of these, 25 signatures are genuine and 25 signatures are skilled forgeries. 5 genuine signatures are selected randomly from genuine signatures to be used as references. The construction and description of DB1 is shown in TABLE I.

TABLE I. DESCRIPTION OF ON-LINE SIGNATURE VERIFICATION DAT ASET

\begin{tabular}{cc}
\hline Descriptions & Signature Details \\
\hline No. of References & $100 * 5=500$ (Genuines) \\
\hline No. of Test Signatures & $10 * 20=2000$ (Genuines) \\
& $10 * 25=2500$ (Skilled \\
forgeries)
\end{tabular}




\section{B. Feature Extraction}

6 features are extracted to be used in our works, i.e. $\mathrm{F}_{-}$Base $=\left\{X, Y, V x, V y, a_{c}, P\right\}$. Out of these, feature $X(n), Y(n)$ and $P(n)$ are obtained directly from signature acquisition devices.

Features of $V x(n), V y(n), a_{c}(n)$ are extracted by simple mathematical computation as,

- Linear Velocity in x-direction: $V_{x}(n)=$ $(X(n+1)-X(n-1)) / 2$;

- Linear Velocity in y-direction: $V_{y}(n)=$ $(Y(n+1)-Y(n-1)) / 2$;

- Centripetal Acceleration: $a_{c}(n)=$ $\left(V_{x}(n) \cdot a_{y}(n)-V_{y}(n) \cdot a_{x}(n)\right) / V(n)$, where, $a_{x}(n)$ and $a_{y}(n)$ is the linear Acceleration in x-direction and y-direction, $\quad a_{x}(n)=\left(V_{x}(n+1)-V_{x}(n-1)\right) / 2$ $a_{y}(n)=\left(V_{y}(n+1)-V_{y}(n-1)\right) / 2, V(n)$ is the absolute velocity, $V(n)=\sqrt{V_{x}(n)^{2}+V_{y}(n)^{2}}$.

\section{Alignment of On-Line Signature}

On-line signature dataset DB1 is used to the experiments of alignment, results of alignment of individual-30 are given in Fig. 1 and Fig. 2. From the Fig. 1, as for genuine signature, the best matching is obtained by centroid alignment, and the worst matching is given by size align ment. While as for skilled forgeries, the same conclusion is obtained from Fig. 2.The experiment results indicate the best matching between test and reference signatures could be obtained by centroid alignment.

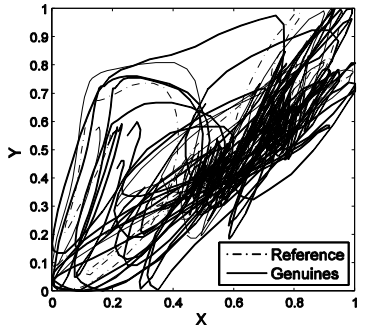

(a)

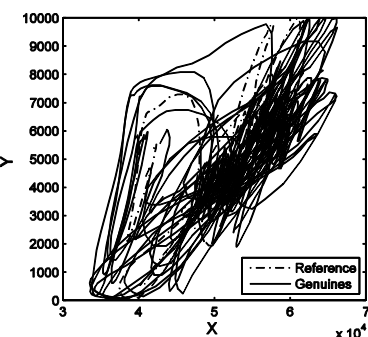

(b)

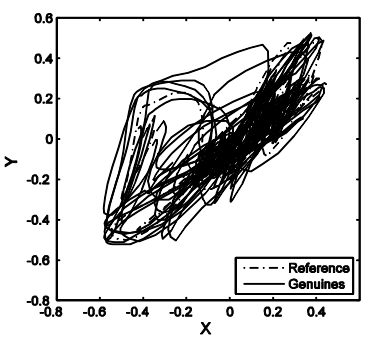

(c)

Figure 1. Alignment between genuine signature and reference.(a)alignment with size,(b)alignment with signature center,(c) alignment with signature centroid

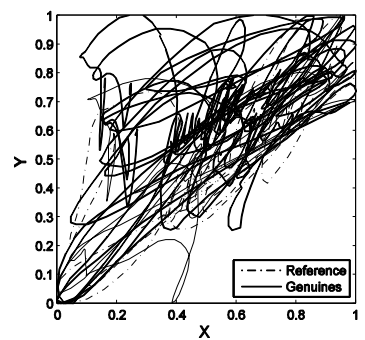

(a)

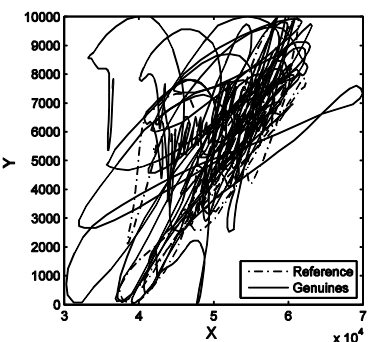

(b)

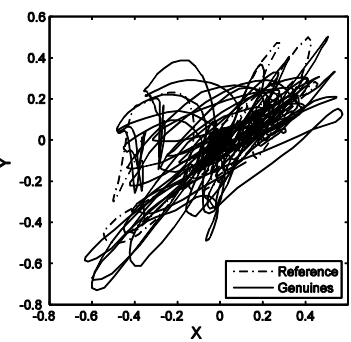

(c)

Figure 2. Alignment between skilled forgeries and reference.(a)alignment with size,(b)alignment with signature center,(c) alignment wit h signature centroid

\section{Results of On-Line Signature Verification}

To verify the effectiveness of our proposed method of signature alignment and similarity calculation, the authenticity of 4500 test signatures from 100 individuals are determined. Error rates of EER(Equal Error Rate), FRR(False Reject Rate),FAR(False Accept Rate) are adopted to evaluate the performance of on-line signature verification, in which EER could indicate the security level of a given biometrics system. Experiment results of different methods of signature alignment are given in TABLE II, which with the traditional DTW as similarity calculation. From the TABLE II, it is obtained lowest $\mathrm{EER}=3.89 \%$ with centroid align ment, which indicates that it could be obtained optimal performance after align ment with signature centroid. Error rates of on-line signature verification with different methods of similarity calculation are shown in TABLE III, with signature centroid alignment. EER of signature verification with SCLC-DTW as similarity calculation is $2.99 \%$, which increase $0.9 \%$ performance compared with traditional DTW. The experiment results indicate the effectiveness of our proposed method.

\begin{tabular}{cccc}
\hline Method of Alignment & FRR $(\boldsymbol{\%})$ & FAR $(\boldsymbol{\%})$ & EER $(\%)$ \\
\hline Size & 5.6 & 6.2 & 5.9 \\
Center & 4 & 5.08 & 4.54 \\
Centroid & 4.7 & 3.08 & $\mathbf{3 . 8 9}$
\end{tabular}



CALCULATION (CENT ROID ALIGNMENT)

\begin{tabular}{cccc}
\hline Method of Verification & FRR (\%) & FAR (\%) & EER (\%) \\
\hline DTW & 4.7 & 3.08 & 3.89 \\
SCLC-DTW & 3.5 & 2.48 & $\mathbf{2 . 9 9}$ \\
\hline
\end{tabular}

\section{CONCLUSIONS}

We propose some methods of alignment to obtain the optimal matching between test and reference signatures. We also propse a new method of similarity calculation based on SCLC-DTW to improve the efficiency of signature verification. Severel experiment results indicate the effectiveness of our proposed methods.

\section{ACKNOWLEDGMENT}

The authors wish to thank Dr. J. Fierrez-Aguilar and M. Go mez-Barrero to sharing the MCYT data set from Biometric Research Lab-AVTS, Madrid, Spain. This work was financially supported by the Natural Science Foundation of China (61305125), Liaoning Province Natural Science Foundation (2013020012).

\section{REFERENCES}

[1] Plamondon R, Srihari S, On-Line and Off-Line Handwriting Recognition: A Comprehensive Survey. IEEE Transactions on Pattern Analysis and Machine Intelligence, 2000, 22(1): 63-84.

[2] Impedovo D, Pirlo G, Automatic Signature Verification: The State of The Art,IEEE Transactions on Systems, Man, and Cybernetics, Part C: Applications and Reviews, 2008, 38(5): 609-635.

[3] Thumwarin, Pitak, Jitawat Pernwong, and Takenobu Matsuura. FIR signature verification system characterizing dynamics of handwriting features[J]. EURASIP Journal on Advances in Signal Processing, 2013, 2013(1): 1-15.

[4] Igarza, Juan J., et al. Searching for an optimal reference system for on-line signature verification based on $(\mathrm{x}, \mathrm{y})$ alignment[J]. Biometric Authentication. Springer Berlin Heidelberg, 2004, 519-525.

[5] Al-Mayyan W, Own H S, Zedan H. Rough set approach to online signature identification[J]. Digital Signal Processing, 2011, 21(3): 477-485.

[6] Lopez-Garcia M, Ramos-Lara R, Miguel-Hurtado O, et al. Embedded System for Biometric Online Signature Verification[J]. Industrial Informatics, IEEE Transactions on, 2014, 10(1): 491-501.

[7] Cpałka K, Zalasiński M. On-line signature verification using vertical signature partitioning[J]. Expert Systems with Applications, 2014, 41(9): 4170-4180.

[8] Reza A G, Lim H, Alam M J. An efficient online signature verification scheme using dynamic programming of string matching $[\mathrm{M}] / /$ Convergence and Hybrid Informat ion Technology. Springer Berlin Heidelberg, 2011:590-597.

[9] Vivaracho-Pascual C, Faundez-Zanuy M, Pascual J M. An Efficient Low Cost Approach for On-Line Signature Recognition Based on Length Normalization and Fractional Distances. Pattern Recognition, 2009, 42(1): 183-193.

[10] Faundez-Zanuy M. On-Line Signature Recognition Based on VQ-DT W. Pattern Recognition, 2007, 40(3): 981-992.

[11] Ortega-Garcia J, Fierrez-Aguilar J, Simon D. MCYT baseline corpus: a bimodal biometric database [J]. IEE ProceedingsVision, Image and Signal Processing, 2003, 150(6): 395-401. 